\title{
Factors explaining firms' receipt of public funding for innovation: the case of Chilean small and medium-sized enterprises ${ }^{\star}$
}

\author{
Cristian Mardones ${ }^{1 * *}$, Annabella Zapata ${ }^{1}$
}

\begin{abstract}
This study uses innovation surveys conducted in Chile to evaluate the factors that may explain public financing for innovative activities in small and medium-sized firms (SMEs). The analysis is important because small and medium sized firms are the focus of the existing public programs. The estimated results with binary choice models are contrasted with cross-sectional and pseudo-panel data, observing that firms with some types of expenditures on innovative activities in previous year have more probability to obtain public financing.
\end{abstract}

Keywords: Pseudo-panel; Probit; Public financing.

Submitted: January $19^{\text {th }}, 2017$ / Approved: July 17 $7^{\text {th }}, 2018$

\section{Introduction}

Empirical evidence shows that R\&D is responsible for growth in productivity. For example, Bravo-Ortega et al. (2014) use cross-sectional data to examine the relation between productivity, expenditure in $\mathrm{R} \& \mathrm{D}$ and exports in Chile, being found that the firms that invest in $\mathrm{R} \& \mathrm{D}$ are more likely to export, $\mathrm{R} \& \mathrm{D}$ improves productivity, and public funding complements private resources for $R \& D$. Álvarez et al. (2010) suggest that in Chile there is no contemporary effect of innovation in products on productivity, though lagged effects are observed after two years. Crespi et al. (2015) use panel data from Colombian firms to demonstrate that the financial incentive programs for $R \& D$ have increased labor productivity. Álvarez et al. (2015) point out that in the case of Chile there is an effect of the technological and nontechnological innovation on labor productivity in the manufacturing and services sectors. More recently, Crespi et al. (2016) demonstrate that the public funding for innovation is a key factor to facilitate investment in innovation in manufacturing firms of Latin America.

R\&D expenditure as a percentage of GDP is low in Chile (0.4\%) compared to OECD countries (2.4\%). Moreover, Cabaleiro \& Salce (2018) conclude that the markets for technology in Chile are not developed. These facts could be explained by uncertain returns from investing in innovation and R\&D which affect the conditions for financing this type of activities. For this reason, in recent years the Chilean State has played a more active role in innovation ${ }^{1}$ through the creation of diverse programs to mitigate the different obstacles and constraints faced by innovative activities in small and medium-sized firms.

The decision to allocate resources to $\mathrm{R} \& \mathrm{D}$ activities is influenced by the characteristics of the firm, the market and the public incentives. According to Shefer \& Frenkel (2005) a 50\% of the variation in $\mathrm{R} \& \mathrm{D}$ expenditure is explained by innovation, economic sector, total sales, export orientation and age of the firm. Barge-Gil \& López (2014) point out that the evidence is mixed for the effects of financial constraints and public funding instruments on R\&D expenditure. González \& Pazó (2008) conclude that public funding fosters the private technological effort of small firms. Clausen (2009) shows that research subsidies stimulate R\&D expenditure, while development subsidies replace that expenditure. Cerulli \& Potí (2012) find a relationship between $R \& D$ expenditure and variables such as the number of employees, percentage of employees with university degree, percentage of sales from exports, capital per employee, cash flow per employee, percentage of liabilities, IPR value when the firm belongs to a foreign group, age and geographical location.

According to the international literature, the factors that affect the probability of obtaining public financing for innovation are varied. Huergo et al. (2016) indicate that the probability of participating in an $R \& D$ loan system increases when a firm has technological profile and sectoral financial constraints. Afcha (2012) demonstrates that the probability of obtaining an R\&D subsidy is increased by cooperation networks, recruitment of newly graduated professionals, $\mathrm{R} \& \mathrm{D}$ expenditures from previous years, number of employees and exports. Duch-Brown et al. (2011) indicate that having prior experience in $\mathrm{R} \& \mathrm{D}$ projects increases the intensity of subsidies. Cantner \& Kösters (2012) demonstrate that the work team and the initial capital of the firm affect the obtaining of public funds.

Previous studies in Chile have focused mainly on the relationship between firms' productivity and innovative variables (Benavente, 2005; Álvarez et al., 2010) and recently on the impact of knowledge obstacles to introduce innovations (Canales \& Álvarez, 2017) but have not analyzed what factors influence the probability of obtaining public financing. Therefore, this study seeks to determine the factors that affect the receipt of public support for innovation in small and medium-sized firms, considering cross-sectional data from the different versions of the Innovation Surveys. Then, these results are contrasted with those obtained from a pseudo-panel methodology that uses the cross-sectional databases together.

(1) Moya \& Molina (2017) show that both innovation and entrepreneurship are important concepts in the policies promoted by the government of Chile.

(1) Department of Industrial Engineering, University of Concepción, Chile.

**Corresponding author: crismardones@udec.cl

${ }^{*}$ This work was supported by CORFO [INNOVA CHILE N 15 PES-44389, 2016] 


\section{Methodology}

\subsection{Data}

The Innovation Survey of Chilean firms have been collected in nine different versions, the first in 1995 and the last in 2014. Figure 1 shows the number of firms surveyed in each version of the survey. From the first to the fourth survey, the number of firms fluctuates between 520 and 900 , while this number grows by over 5600 in the most recent survey. However, in the last five surveys only $55 \%$ of firms are SMEs.

Figure 1. Number of firms surveyed in each version of the Innovation Survey

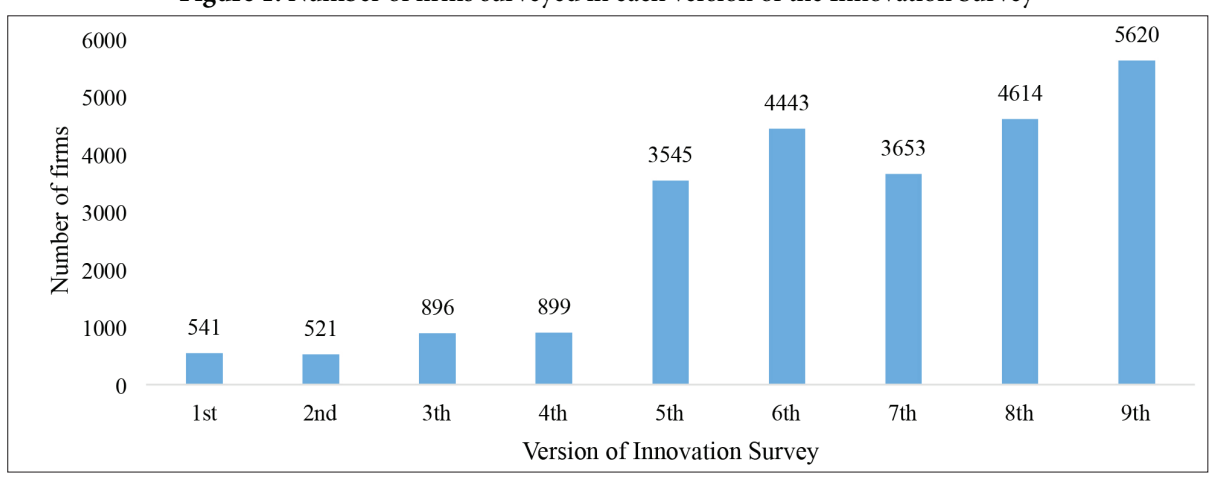

Source: Own elaboration based on data from the 1st to 9th Innovation Surveys

The nine versions of this survey have different structures and variables due to changes in its design but there is greater uniformity starting with the fifth version. Table 1 exemplifies this situation with an extract of some variables that are present in the different versions of the survey.
Therefore, firm-level observations from the fifth to the ninth version of the survey were consolidated into a single database to enable statistical analysis based on cross-sectional estimates and to facilitate a comparison of the results with the pseudo-panel methodology.

Table 1. Extract list of variables database from Innovation Survey

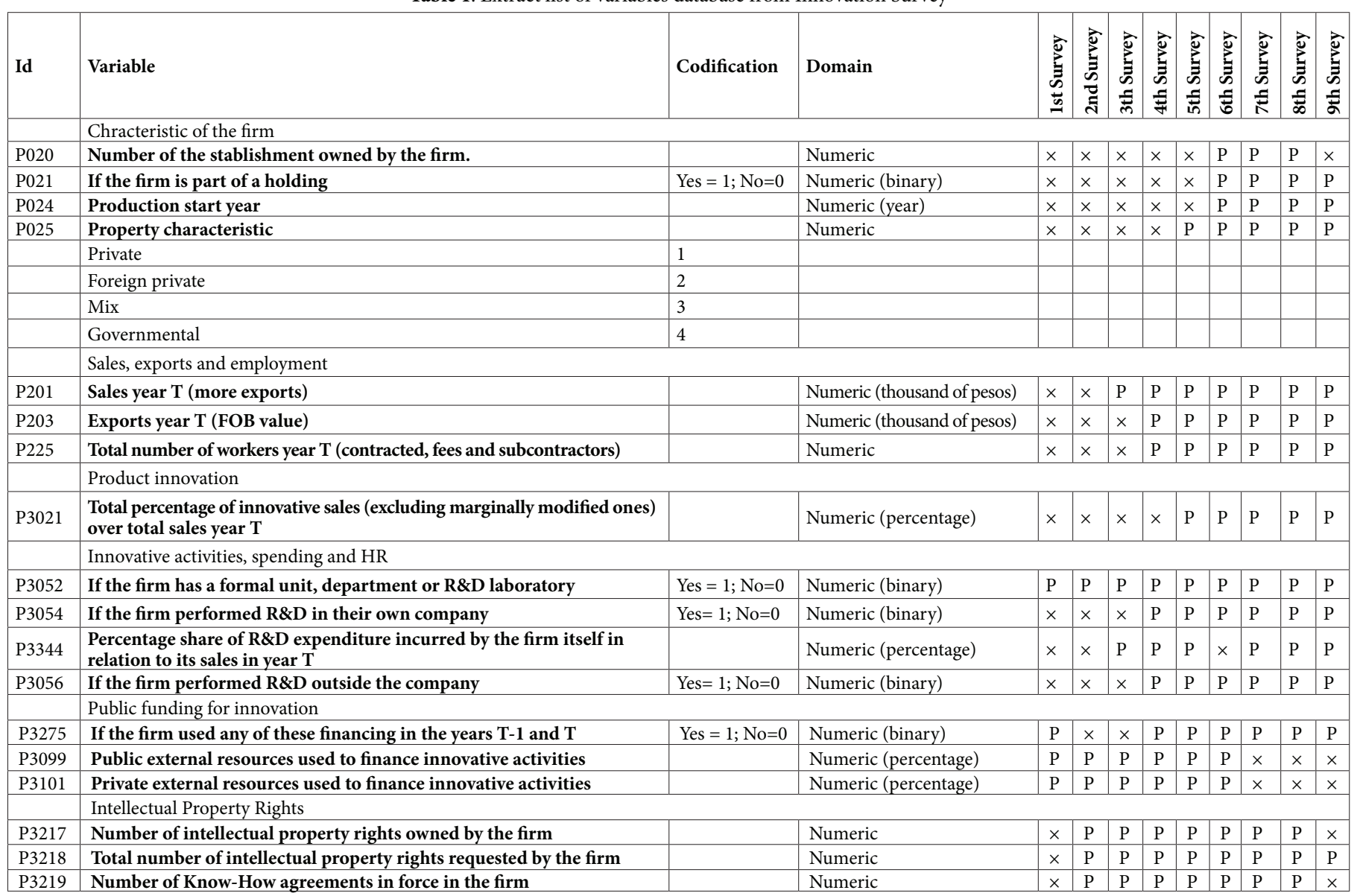

Source: Own elaboration from data obtained from 1st to 9th Innovation Surveys. 


\subsection{Pseudo-panel data}

A typical panel data regression can be represented as:

$$
\begin{gathered}
y_{i t}=\alpha_{i}+x_{i t}^{\prime} \beta+u_{i t} ; \quad i=1, \ldots, N ; \quad t=1, \ldots, T \\
u_{i t}=\mu_{i}+v_{i t}
\end{gathered}
$$

Where subscript $i$ indicates the cross-sectional dimension that can denote, for example, individuals, families, firms, and countries, and $t$ indicates the time. In this model, $y_{i t}$ it is the dependent variable and $x_{i t}$ it is a vector of $K$ explanatory variables. In addition, most panel data applications use a model with two error components, $\mu_{i}$ denoting the individual non-observable effect and $v_{i t}$ denoting the remaining disturbance.

Although in many developing countries there is little availability of panel data, it is possible to have repeated cross-sectional data in which the same individuals are not tracked over time. Under this focus, individuals share some common characteristics (in this study, firms belonging to the same economic sector), whereby they can be grouped into cohorts and the averages within the cohorts are treated as observations in a pseudo-panel.

Deaton (1985) suggests the use of cohorts to obtain consistent estimators for $\beta$ in (1), even if the individual effects $\alpha_{i}$ are correlated with explanatory variables. By defining $C$ cohorts in which each individual is a member of a single cohort for all periods, all the observations are grouped at the cohort level, so the resulting model can be written as:

$$
\bar{y}_{c t}=\bar{x}_{c t}^{\prime} \beta+\bar{\alpha}_{c t}+\bar{u}_{c t} ; \quad c=1, \ldots, C ; t=1, \ldots, T
$$

Where $\bar{y}_{c t}$ is the average value of all $y_{i t}$ in cohort $c$ at time $t$. This is analogously the case for the other variables in the model. The resulting dataset is a pseudo-panel with repeated observations over $T$ periods and $C$ cohorts.

Subsequently, Moffitt (1993) proposes estimating pseudo-panel data through instrumental variables. The interpretation of instrumental variables is useful because it illustrates that alternative estimators can be constructed using other sets of instruments.

\subsection{Binary models}

Binary choice models such as probit or logit are widely known and used in empirical applications with cross-sectional data, whereas with data panel the models typically used are probit random effects and logit fixed effects. However, binary choice models can also be estimated with pseudo-panels data (Verbeek \& Vella, 2005; Verbeek, 2008). Specifically, the binary choice model using pseudo-panel data with instrumental variables proposed by Moffitt (1993) can be written as:

$$
\begin{gathered}
y_{i t}^{*}=x_{i t}^{\prime} \beta+\alpha_{i}+u_{i t}, \\
y_{i t}= \begin{cases}1, & \text { if } y_{i t}^{*} \geq 0, \\
0, & \text { in other case }\end{cases}
\end{gathered}
$$

In this case, the dependent variable $y_{i t}^{*}$ is not observed, but the binary variable $y_{i t}$ is observed and defined by $y_{i t}=1$ (if $y_{i t}^{*}>0$ ) or 0 otherwise. This approach uses dummy variables from the cohorts as instruments for the explanatory variables. Specifically, each individual effect $\alpha_{i}$ is decomposed into a cohort effect $\alpha_{c}$ and the deviation of individual $i$ from this effect. It could be defined as $(c=1, \ldots, C)$ if individual $i$ is a member of cohort $C$, and as 0 otherwise. Thus, $\alpha_{i}$ can be rewritten as:

$$
\alpha_{i}=\sum_{c=1}^{c} \alpha_{c} z_{c i}+\varepsilon_{i}
$$

Defining $\alpha=\left(\alpha_{1}, \ldots, \alpha_{c}\right)^{\prime}$ and $z_{i}=\left(z_{1 i}, \ldots, z_{c i}\right)^{\prime}$, and then substituting (6) into (4) produces the following:

$$
y_{i t}^{*}=x_{i t}^{\prime} \beta+z_{i}^{\prime} \alpha+\varepsilon_{i}+u_{i t} ; \quad t=1, \ldots, T
$$

The next step is to choose the dummy cohort variables in $z_{i}$, interacted in time as instruments, in which case linear predictors are:

$$
x_{k, i t}=z_{i}^{\prime} \delta_{k t}+w_{k, i t} ; \quad k=1, \ldots, K ; t=1, \ldots, T
$$

Where $\delta_{k t}$ is a vector of unknown parameters. The linear predictor for $x_{i t}$ is given by $\hat{x}_{i t}=\bar{x}_{c t}$, the vector of means in cohort $c$ in period $t$. In addition, if it is assumed that $\varepsilon_{i}+u_{i t}$ has a normal distribution and that the instruments for $x_{i t}$ are not correlated with $\varepsilon_{i}+u_{i t}$. Under these assumptions, the instrumental variable estimator produces a consistent estimator for $\beta$ and $\alpha_{c}$.

\section{Results}

The study seeks to evaluate whether a firm's innovative actions carried out in the previous year, as well as other characteristics, affect the probability of receiving public support in the current period. This information is relevant for small and medium-sized firms because they may wish to alter their decisions before applying for these types of funds to increase their chances of obtaining them.

To determine the robustness of the results, several specifications of the empirical model are included. Model 1 only includes, as explanatory variables, expenditures on innovative actions carried out in the previous period. Model 2 controls by total sales, number of workers and whether the firm had exports in the previous period; Model 3 also controls by economic sector; Model 4 also adds a control by region of the firm's location; Model 5 controls by size of the firm and model 6 by type of ownership.

Table 2, Table 3, Table 4, Table 5 and Table 6 present the results obtained from probit models that estimate the probability of obtaining public financing using cross-sectional data from the fifth to the ninth version of the Survey of Innovation, respectively.

Table 2 shows that the expenditure on external knowledge in previous year and exports in previous year have a positive, significant and robust effect on the probability of obtaining public financing. On the other hand, there is a positive, significant and robust effect between specifications for firms located in the Antofagasta Region, Coquimbo Region, Valparaíso Region, O’Higgins Region, Bío Bío Region, Los Lagos Region and Metropolitan Region. However, there is also a significant, robust and negative effect for the firms have private and foreign property. 
Table 2. Results with data from the fifth innovation survey

\begin{tabular}{|c|c|c|c|c|c|c|c|c|c|c|c|c|c|c|c|c|c|c|}
\hline & Model 1 & & & Model 2 & & & Model 3 & & & Model 4 & & & Model 5 & & & Model 6 & & \\
\hline & Coef. & St. Error & & Coef. & St. Error & & Coef. & St. Error & & Coef. & $\begin{array}{l}\text { St. } \\
\text { Error }\end{array}$ & & Coef. & St. Error & & Coef. & St. Error & \\
\hline $\begin{array}{l}\text { Expenditure on equipment for } \\
\text { innovation in } t-1\end{array}$ & $4.2 \mathrm{E}-07$ & $8.9 \mathrm{E}-07$ & & $5.7 \mathrm{E}-07$ & 9E-07 & & $1 \mathrm{E}-06$ & $9.3 \mathrm{E}-07$ & & $1.2 \mathrm{E}-06$ & $9.2 \mathrm{E}-07$ & & $1.1 \mathrm{E}-06$ & $9.3 \mathrm{E}-07$ & & $1.2 \mathrm{E}-06$ & $9.3 \mathrm{E}-07$ & \\
\hline $\begin{array}{l}\text { Expenditure on external } \\
\text { knowledge in } t-1\end{array}$ & $8.4 \mathrm{E}-06$ & $2.7 \mathrm{E}-06$ & $* *$ & $8.1 \mathrm{E}-06$ & $2.8 \mathrm{E}-06$ & $* *$ & 7.1E-06 & $2.6 \mathrm{E}-06$ & $* *$ & 7.1E-06 & $2.7 \mathrm{E}-06$ & $* *$ & $6.9 \mathrm{E}-06$ & $2.6 \mathrm{E}-06$ & $* *$ & $8.4 \mathrm{E}-06$ & $3 \mathrm{E}-06$ & ** \\
\hline $\begin{array}{l}\text { Expenditure on training for } \\
\text { innovation in } t-1\end{array}$ & $4.5 \mathrm{E}-06$ & $3.8 \mathrm{E}-06$ & & $4.4 \mathrm{E}-06$ & $4 \mathrm{E}-06$ & & $2.4 \mathrm{E}-06$ & 4.1E-06 & & $2.8 \mathrm{E}-06$ & $4.4 \mathrm{E}-06$ & & $3.2 \mathrm{E}-06$ & $4.4 \mathrm{E}-06$ & & $3.1 \mathrm{E}-06$ & $4.6 \mathrm{E}-06$ & \\
\hline $\begin{array}{l}\text { Expense on introd. of innova- } \\
\text { tions to the market } t-1\end{array}$ & $3.8 \mathrm{E}-06$ & $2.4 \mathrm{E}-06$ & & 3.7E-06 & $2.5 \mathrm{E}-06$ & & $4.8 \mathrm{E}-06$ & $2.6 \mathrm{E}-06$ & & $3.5 \mathrm{E}-06$ & $2.8 \mathrm{E}-06$ & & $3.5 \mathrm{E}-06$ & $2.8 \mathrm{E}-06$ & & $3.7 \mathrm{E}-06$ & $2.8 \mathrm{E}-06$ & \\
\hline $\begin{array}{l}\text { Expenditure on other innova- } \\
\text { tion activities in } t-1\end{array}$ & $4.8 \mathrm{E}-06$ & $2.8 \mathrm{E}-06$ & & $3.8 \mathrm{E}-06$ & $2.7 \mathrm{E}-06$ & & $3 \mathrm{E}-06$ & $2.8 \mathrm{E}-06$ & & $2.1 \mathrm{E}-06$ & $3 \mathrm{E}-06$ & & $1.7 \mathrm{E}-06$ & $3.1 \mathrm{E}-06$ & & $9.2 \mathrm{E}-07$ & $3.2 \mathrm{E}-06$ & \\
\hline $\begin{array}{l}\text { No. of Intellectual property } \\
\text { rights in } t-1\end{array}$ & 0.00188 & 0.00589 & & -0.0003 & 0.00602 & & 0.00054 & 0.00615 & & -0.0003 & 0.00701 & & 0.00025 & 0.00693 & & -0.0008 & 0.00718 & \\
\hline Total sales in $t-1$ & & & & $3.9 \mathrm{E}-09$ & $6.1 \mathrm{E}-09$ & & $4.4 \mathrm{E}-09$ & 8.9E-09 & & $9.6 \mathrm{E}-09$ & $4.6 \mathrm{E}-08$ & & $3.9 \mathrm{E}-09$ & $5.4 \mathrm{E}-09$ & & $3.9 \mathrm{E}-09$ & $5.6 \mathrm{E}-09$ & \\
\hline Number of workers in $t-1$ & & & & -0.0002 & 0.00069 & & -0.0006 & 0.00085 & & -0.0002 & 0.00083 & & -0.0005 & 0.00091 & & -0.0008 & 0.00094 & \\
\hline Exports in $t-1$ & & & & 0.75 & 0.125 & ** & 0.69 & 0.133 & $* *$ & 0.62 & 0.142 & $* *$ & 0.578 & 0.143 & ** & 0.596 & 0.144 & $* *$ \\
\hline Agricultural and forestry sector & & & & & & & 3.424 & 138.2 & & 5.359 & 1933.4 & & 4.239 & 102.6 & & 4.634 & 132.1 & \\
\hline Fishing sector & & & & & & & 3.175 & 138.2 & & 5.261 & 1933.4 & & 4.123 & 102.6 & & 4.524 & 132.1 & \\
\hline Mining sector & & & & & & & 3.675 & 138.2 & & 4.881 & 1933.4 & & 3.672 & 102.6 & & 4.099 & 132.1 & \\
\hline Manufacturing sector & & & & & & & 2.713 & 138.2 & & 3.832 & 1933.4 & & 2.657 & 102.6 & & 3.025 & 132.1 & \\
\hline Electricity, gas and water sector & & & & & & & 2.961 & 138.2 & & 4.241 & 1933.4 & & 3.057 & 102.6 & & 3.373 & 132.1 & \\
\hline Construction sector & & & & & & & 2.596 & 138.2 & & 4.685 & 1933.4 & & 3.515 & 102.6 & & 3.89 & 132.1 & \\
\hline Commerce sector & & & & & & & 3.269 & 138.2 & & 4.408 & 1933.4 & & 3.234 & 102.6 & & 3.613 & 132.1 & \\
\hline Transport sector & & & & & & & 2.412 & 138.2 & & 4.475 & 1933.4 & & 3.339 & 102.6 & & 3.707 & 132.1 & \\
\hline Financial Services sector & & & & & & & 2.705 & 138.2 & & 4.904 & 1933.4 & & 3.746 & 102.6 & & 4.112 & 132.1 & \\
\hline Real estate sector & & & & & & & 3.458 & 138.2 & & 5.415 & 1933.4 & & 4.268 & 102.6 & & 4.619 & 132.1 & \\
\hline $\begin{array}{l}\text { Social and health services } \\
\text { sector }\end{array}$ & & & & & & & 2.487 & 138.2 & & 4.657 & 1933.4 & & 3.54 & 102.6 & & 3.895 & 132.1 & \\
\hline Other sectors & & & & & & & 2.688 & 138.2 & & 4.682 & 1933.4 & & 3.542 & 102.6 & & 3.89 & 132.1 & \\
\hline Tarapacá Region & & & & & & & & & & 0.835 & 0.49 & & 0.835 & 0.5 & & 0.828 & 0.502 & \\
\hline Antofagasta Region & & & & & & & & & & 0.98 & 0.463 & * & 0.965 & 0.468 & ${ }^{*}$ & 0.953 & 0.469 & * \\
\hline Atacama Region & & & & & & & & & & 0.772 & 0.68 & & 0.791 & 0.692 & & 0.52 & 0.737 & \\
\hline Coquimbo Region & & & & & & & & & & 1.17 & 0.384 & $* *$ & 1.157 & 0.386 & ** & 1.099 & 0.389 & $*$ \\
\hline Valparaíso Region & & & & & & & & & & 1.168 & 0.288 & $* *$ & 1.199 & 0.289 & $* *$ & 1.179 & 0.29 & $* *$ \\
\hline O’Higgins Region & & & & & & & & & & 1.461 & 0.363 & $* *$ & 1.5 & 0.362 & ** & 1.49 & 0.363 & $*$ \\
\hline Maule Region & & & & & & & & & & . & . & & . & . & & . & . & \\
\hline Bío Bío Region & & & & & & & & & & 1.256 & 0.297 & $* *$ & 1.289 & 0.299 & $*$ & 1.296 & 0.3 & $*$ \\
\hline Araucania Region & & & & & & & & & & . & . & & . & . & & . & . & \\
\hline Los Lagos Region & & & & & & & & & & 1.195 & 0.303 & $* *$ & 1.175 & 0.305 & $*$ & 1.171 & 0.307 & $*$ \\
\hline Aysén Region & & & & & & & & & & . & . & & . & . & & . & . & \\
\hline Magallanes Region & & & & & & & & & & . & $\cdot$ & & . & . & & . & . & \\
\hline Metropolitan Region & & & & & & & & & & 1.176 & 0.197 & * & 1.184 & 0.197 & $*$ & 1.171 & 0.199 & $* *$ \\
\hline Los Rios Region & & & & & & & & & & . & . & & . & . & & & & \\
\hline Small firm & & & & & & & & & & & & & -0.219 & 0.122 & & -0.24 & 0.124 & \\
\hline Private property & & & & & & & & & & & & & & & & -1.22 & 0.558 & * \\
\hline Foreign Property & & & & & & & & & & & & & & & & -1.918 & 0.768 & * \\
\hline Mixed Property & & & & & & & & & & & & & & & & -0.916 & 0.672 & \\
\hline Constant & -1.762 & 0.0513 & $*$ & -1.892 & 0.0631 & $* *$ & -4.823 & 138.2 & & -7.041 & 1933.4 & & -5.757 & 102.6 & & -4.89 & 132.1 & \\
\hline Observations & 2048 & & & 2048 & & & 2048 & & & 1909 & & & 1909 & & & 1909 & & \\
\hline Pseudo R2 & 0.036 & & & 0.09 & & & 0.157 & & & 0.215 & & & 0.219 & & & 0.229 & & \\
\hline
\end{tabular}

Source: Own elaboration. Note: $\left({ }^{*}\right)$ significant at $5 \%$ and $\left(^{* *}\right)$ significant at $1 \%$. 
Table 3 shows that the expenditure on external knowledge, expenditure on introduction of innovations to the market, and exports in previous year have a positive, significant and robust effect on the probability of obtaining public financing. At sectoral level, it is observed that the real state sector has a positive, significant and robust effect.

Table 3. Results with data from the sixth innovation survey

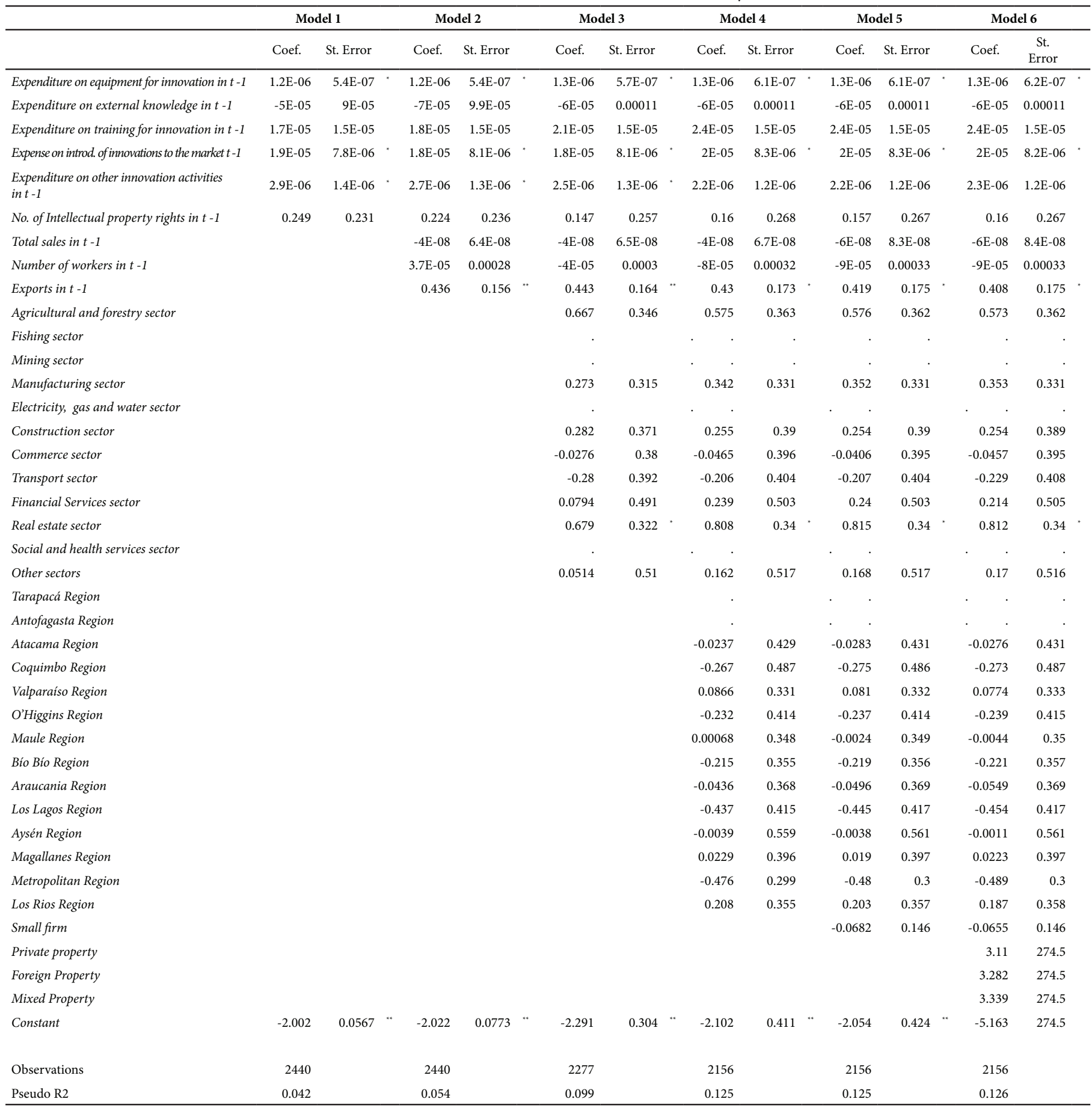

Source: Own elaboration. Note: $\left(^{*}\right)$ significant at $5 \%$ and $\left({ }^{* *}\right)$ significant at $1 \%$.

Table 4 shows that there are no statistically significant and robust effects of expenditures on innovative activities carried out in the previous year on the probability of obtaining public support. However, there is also a significant, robust and negative effect for small sized and private property firms. 
Table 4. Results with data from the seventh innovation survey

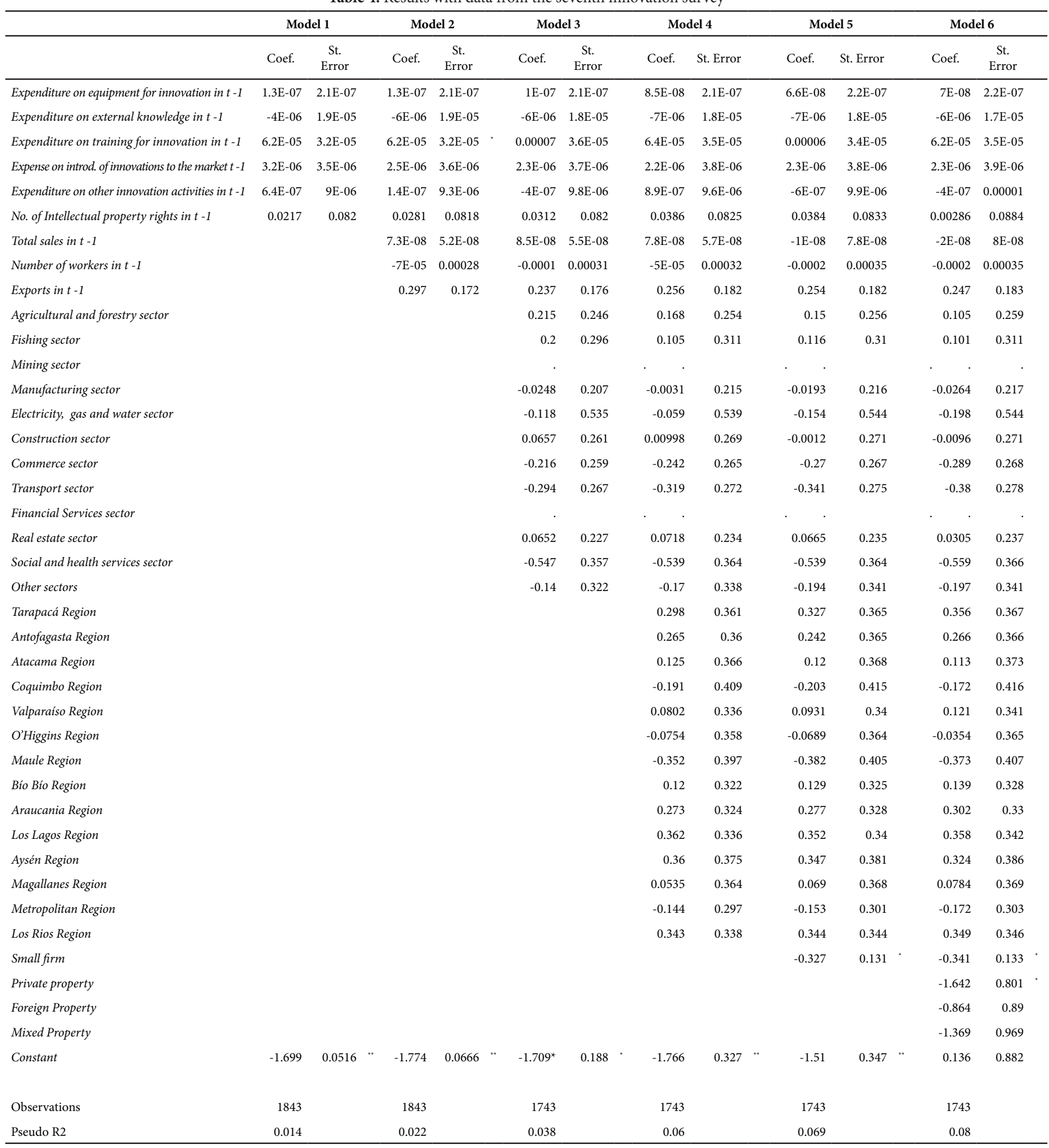

Source: Own elaboration. Note: $\left(^{*}\right)$ significant at $5 \%$ and $\left({ }^{* *}\right)$ significant at $1 \%$.

Table 5 shows that the expenditure on other innovation activities in previous year and exports in previous year have a positive, significant and robust effect on the probability of obtaining public financing. On the other hand, there is a negative, significant and robust effect between specifications for firms located in the Valparaíso Region and O'Higgins Region. However, there is also a significant, robust and negative effect for small sized firms and foreign property firms. 
Table 5. Results with data from the eighth innovation survey

\begin{tabular}{|c|c|c|c|c|c|c|c|c|c|c|c|c|c|c|c|c|c|c|}
\hline & \multicolumn{2}{|c|}{ Model 1} & & \multicolumn{3}{|c|}{ Model 2} & \multicolumn{2}{|c|}{ Model 3} & \multicolumn{4}{|c|}{ Model 4} & \multicolumn{2}{|c|}{ Model 5} & \multicolumn{4}{|c|}{ Model 6} \\
\hline & Coef. & $\begin{array}{l}\text { St. } \\
\text { Error }\end{array}$ & & Coef. & St. Error & & Coef. & St. Error & & Coef. & St. Error & & Coef. & $\begin{array}{l}\text { St. } \\
\text { Error }\end{array}$ & & Coef. & $\begin{array}{l}\text { St. } \\
\text { Error }\end{array}$ & \\
\hline Expenditure on equipment for innovation in $t-1$ & 4.06E-08 & $1.1 \mathrm{E}-07$ & & 4.41E-08 & $1.1 \mathrm{E}-07$ & & $5.59 \mathrm{E}-08$ & $1.1 \mathrm{E}-07$ & & $5.67 \mathrm{E}-08$ & $1.1 \mathrm{E}-07$ & & $\begin{array}{r}4.56 \mathrm{E}- \\
08\end{array}$ & $1.1 \mathrm{E}-07$ & & $\begin{array}{r}3.98 \mathrm{E}- \\
08\end{array}$ & $1.2 \mathrm{E}-07$ & \\
\hline Expenditure on external knowledge in $t-1$ & $2.8 \mathrm{E}-06$ & $5.3 \mathrm{E}-06$ & & $3.7 \mathrm{E}-06$ & $5.1 \mathrm{E}-06$ & & $4 \mathrm{E}-06$ & $5.2 \mathrm{E}-06$ & & 4.2E-06 & $5.3 \mathrm{E}-06$ & & $3.8 \mathrm{E}-06$ & $5.6 \mathrm{E}-06$ & & $3.8 \mathrm{E}-06$ & $5.6 \mathrm{E}-06$ & \\
\hline Expenditure on training for innovation in $t-1$ & $-3 \mathrm{E}-07$ & $2.6 \mathrm{E}-06$ & & $-6 \mathrm{E}-07$ & $2.7 \mathrm{E}-06$ & & $-9 \mathrm{E}-07$ & $2.8 \mathrm{E}-06$ & & $-1 \mathrm{E}-06$ & $2.9 \mathrm{E}-06$ & & $-1 \mathrm{E}-06$ & $3 \mathrm{E}-06$ & & $-1 \mathrm{E}-06$ & $3.6 \mathrm{E}-06$ & \\
\hline $\begin{array}{l}\text { Expense on introd. of innovations to the } \\
\text { market } t-1\end{array}$ & $-2 \mathrm{E}-06$ & $1.1 \mathrm{E}-05$ & & $-1 \mathrm{E}-06$ & $1.2 \mathrm{E}-05$ & & $-4 \mathrm{E}-06$ & $1.2 \mathrm{E}-05$ & & $-2 \mathrm{E}-06$ & $1.3 \mathrm{E}-05$ & & $-2 \mathrm{E}-06$ & $1.4 \mathrm{E}-05$ & & $-1 \mathrm{E}-06$ & $1.3 \mathrm{E}-05$ & \\
\hline $\begin{array}{l}\text { Expenditure on other innovation activities } \\
\text { in } t-1\end{array}$ & $8.9 \mathrm{E}-06$ & $2.6 \mathrm{E}-06$ & ** & $9.6 \mathrm{E}-06$ & $3.2 \mathrm{E}-06$ & $*$ & $1 \mathrm{E}-05$ & $3.3 \mathrm{E}-06$ & $* *$ & $1 \mathrm{E}-05$ & $3.4 \mathrm{E}-06$ & ${ }^{* *}$ & $1 \mathrm{E}-05$ & $3.5 \mathrm{E}-06$ & $* *$ & 9E-06 & $3.5 \mathrm{E}-06$ & * \\
\hline No. of Intellectual property rights in $t-1$ & 0.0227 & 0.0274 & & 0.0121 & 0.028 & & 0.0146 & 0.0292 & & 0.015 & 0.0295 & & 0.0163 & 0.0304 & & 0.0191 & 0.0296 & \\
\hline Total sales in $t-1$ & & & & $-7 \mathrm{E}-08$ & $6.7 \mathrm{E}-08$ & & $-7 \mathrm{E}-08$ & $6.7 \mathrm{E}-08$ & & $-8 \mathrm{E}-08$ & $6.9 \mathrm{E}-08$ & & $-2 \mathrm{E}-07$ & $1 \mathrm{E}-07$ & * & $-2 \mathrm{E}-07$ & $1 \mathrm{E}-07$ & * \\
\hline Number of workers in $t-1$ & & & & -0.0004 & 0.00066 & & -0.0004 & 0.00067 & & -0.0004 & 0.00066 & & -0.0005 & 0.00069 & & -0.0009 & 0.00079 & \\
\hline Exports in $t-1$ & & & & 0.539 & 0.149 & $*$ & 0.568 & 0.155 & $* *$ & 0.573 & 0.16 & $* *$ & 0.543 & 0.161 & $* *$ & 0.55 & 0.164 & $*$ \\
\hline Agricultural and forestry sector & & & & & & & 0.514 & 0.274 & & 0.555 & 0.283 & $*$ & 0.584 & 0.284 & $*$ & 0.576 & 0.284 & * \\
\hline Fishing sector & & & & & & & 0.182 & 0.352 & & 0.226 & 0.365 & & 0.288 & 0.367 & & 0.287 & 0.367 & \\
\hline Mining sector & & & & & & & & . & & . & . & & . & . & & . & . & \\
\hline Manufacturing sector & & & & & & & -0.0509 & 0.245 & & -0.0297 & 0.254 & & -0.0223 & 0.255 & & -0.0374 & 0.255 & \\
\hline Electricity, gas and water sector & & & & & & & . & & & . & $\cdot$ & & . & . & & . & . & \\
\hline Construction sector & & & & & & & 0.372 & 0.279 & & 0.404 & 0.289 & & 0.412 & 0.29 & & 0.419 & 0.29 & \\
\hline Commerce sector & & & & & & & 0.219 & 0.269 & & 0.227 & 0.279 & & 0.256 & 0.279 & & 0.244 & 0.279 & \\
\hline Transport sector & & & & & & & 0.0737 & 0.287 & & 0.124 & 0.297 & & 0.119 & 0.299 & & 0.118 & 0.299 & \\
\hline Financial Services sector & & & & & & & -0.282 & 0.455 & & -0.221 & 0.463 & & -0.182 & 0.461 & & -0.193 & 0.461 & \\
\hline Real estate sector & & & & & & & 0.411 & 0.242 & & 0.456 & 0.25 & & 0.468 & 0.251 & & 0.454 & 0.252 & \\
\hline Social and health services sector & & & & & & & -0.264 & 0.389 & & -0.247 & 0.392 & & -0.24 & 0.393 & & -0.297 & 0.399 & \\
\hline Other sectors & & & & & & & -0.0951 & 0.334 & & -0.0716 & 0.34 & & -0.0607 & 0.341 & & -0.0712 & 0.342 & \\
\hline Tarapacá Region & & & & & & & & & & -0.392 & 0.352 & & -0.397 & 0.354 & & -0.384 & 0.356 & \\
\hline Antofagasta Region & & & & & & & & & & -0.414 & 0.349 & & -0.442 & 0.35 & & -0.433 & 0.352 & \\
\hline Atacama Region & & & & & & & & & & -0.477 & 0.384 & & -0.489 & 0.383 & & -0.475 & 0.384 & \\
\hline Coquimbo Region & & & & & & & & & & -0.253 & 0.332 & & -0.301 & 0.334 & & -0.283 & 0.336 & \\
\hline Valparaíso Region & & & & & & & & & & -1.16 & 0.413 & $*$ & -1.21 & 0.416 & $*$ & -1.194 & 0.418 & * \\
\hline O'Higgins Region & & & & & & & & & & -1.005 & 0.438 & * & -1.05 & 0.435 & * & -1.03 & 0.436 & * \\
\hline Maule Region & & & & & & & & & & -0.417 & 0.325 & & -0.459 & 0.327 & & -0.436 & 0.329 & \\
\hline Bío Bío Region & & & & & & & & & & -0.382 & 0.284 & & -0.404 & 0.284 & & -0.385 & 0.286 & \\
\hline Araucania Region & & & & & & & & & & -0.173 & 0.295 & & -0.197 & 0.295 & & -0.167 & 0.297 & \\
\hline Los Lagos Region & & & & & & & & & & -0.102 & 0.275 & & -0.157 & 0.276 & & -0.127 & 0.278 & \\
\hline Aysén Region & & & & & & & & & & 0.476 & 0.341 & & 0.443 & 0.341 & & 0.468 & 0.344 & \\
\hline Magallanes Region & & & & & & & & & & -0.368 & 0.361 & & -0.357 & 0.361 & & -0.333 & 0.363 & \\
\hline Metropolitan Region & & & & & & & & & & -0.417 & 0.235 & & -0.459 & 0.236 & & -0.449 & 0.238 & \\
\hline Los Rios Region & & & & & & & & & & -0.207 & 0.339 & & -0.249 & 0.344 & & -0.235 & 0.346 & \\
\hline Small firm & & & & & & & & & & & & & -0.344 & 0.129 & ** & -0.355 & 0.13 & * \\
\hline Private property & & & & & & & & & & & & & & & & -1.149 & 0.608 & \\
\hline Foreign Property & & & & & & & & & & & & & & & & -1.563 & 0.774 & ${ }^{*}$ \\
\hline Mixed Property & & & & & & & & & & & & & & & & -0.742 & 0.756 & \\
\hline Constant & -1.725 & 0.0455 & $*$ & -1.716 & 0.0624 & $*$ & -1.883 & 0.227 & $*$ & -1.543 & 0.309 & ${ }^{* *}$ & -1.223 & 0.332 & ${ }^{* *}$ & -0.0667 & 0.706 & \\
\hline Observations & 2428 & & & 2428 & & & 2406 & & & 2406 & & & 2406 & & & 2406 & & \\
\hline Pseudo R2 & 0.022 & & & 0.037 & & & 0.066 & & & 0.099 & & & 0.107 & & & 0.113 & & \\
\hline
\end{tabular}

Source: Own elaboration. Note: $\left(^{*}\right)$ significant at $5 \%$ and $\left(^{* *}\right)$ significant at $1 \%$.

Table 6 shows that the expenditure on training for innovation and number of intellectual property rights in previous year have a positive, significant and robust effect on the probability of obtaining public financing. Moreover, there is also a significant, robust and negative effect of total sales in previous year. At sectoral level, it is observed that the manufacturing, commerce and transport sector have a negative, significant and robust effect. 
Table 6. Results with data from the ninth innovation survey

\begin{tabular}{|c|c|c|c|c|c|c|c|c|c|c|c|c|c|c|c|c|c|}
\hline & \multicolumn{2}{|c|}{ Model 1} & & \multicolumn{3}{|c|}{ Model 2} & \multicolumn{2}{|c|}{ Model 3} & & \multicolumn{2}{|c|}{ Model 4} & & \multicolumn{2}{|c|}{ Model 5} & & \multicolumn{2}{|c|}{ Model 6} \\
\hline & Coef. & St. Error & & Coef. & St. Error & & Coef. & St. Error & & Coef. & St. Error & & Coef. & $\begin{array}{c}\text { St. } \\
\text { Error }\end{array}$ & & Coef. & St. Error \\
\hline Expenditure on equipment for innovation in $t-1$ & $9,3 \mathrm{E}-07$ & $6,2 \mathrm{E}-07$ & & $2,8 \mathrm{E}-06$ & $9,4 \mathrm{E}-07$ & " & $2,6 \mathrm{E}-06$ & $9,6 \mathrm{E}-07$ & " & $2,7 \mathrm{E}-06$ & $9,7 \mathrm{E}-07$ & & $2,9 \mathrm{E}-06$ & $1,1 \mathrm{E}-06$ & " & $2,9 \mathrm{E}-06$ & $1,1 \mathrm{E}-06$ \\
\hline Expenditure on external knowledge in $t-1$ & $4,3 \mathrm{E}-05$ & $2,6 \mathrm{E}-05$ & & $4,2 \mathrm{E}-05$ & $2,6 \mathrm{E}-05$ & & $4,2 \mathrm{E}-05$ & 2,7E-05 & & $4,1 \mathrm{E}-05$ & $2,7 \mathrm{E}-05$ & & $4,2 \mathrm{E}-05$ & $2,7 \mathrm{E}-05$ & & $4,1 \mathrm{E}-05$ & $2,7 \mathrm{E}-05$ \\
\hline Expenditure on training for innovation in $t-1$ & 7E-05 & $1,9 \mathrm{E}-05$ & $*$ & 7,7E-05 & $2 \mathrm{E}-05$ & $*$ & $7,8 \mathrm{E}-05$ & $2,1 \mathrm{E}-05$ & "* & $7,4 \mathrm{E}-05$ & 2,1E-05 & " & $7,4 \mathrm{E}-05$ & $2,1 \mathrm{E}-05$ & $"$ & $7,3 \mathrm{E}-05$ & $2,2 \mathrm{E}-05$ \\
\hline Expense on introd. of innovations to the market $t-1$ & $5 \mathrm{E}-06$ & $1,7 \mathrm{E}-05$ & & $7,6 \mathrm{E}-06$ & $1,8 \mathrm{E}-05$ & & $8,1 \mathrm{E}-06$ & $1,8 \mathrm{E}-05$ & & $9,2 \mathrm{E}-06$ & $1,8 \mathrm{E}-05$ & & 9,1E-06 & $1,8 \mathrm{E}-05$ & & $9,1 \mathrm{E}-06$ & $1,8 \mathrm{E}-05$ \\
\hline Expenditure on other innovation activities in $t-1$ & $1,6 \mathrm{E}-05$ & $8,4 \mathrm{E}-06$ & & $1,7 \mathrm{E}-05$ & $8,6 \mathrm{E}-06$ & . & $1,5 \mathrm{E}-05$ & $8,6 \mathrm{E}-06$ & & $1,4 \mathrm{E}-05$ & $8,7 \mathrm{E}-06$ & & $1,4 \mathrm{E}-05$ & $8,8 \mathrm{E}-06$ & & $1,4 \mathrm{E}-05$ & $8,8 \mathrm{E}-06$ \\
\hline No. of Intellectual property rights in $t-1$ & 0,211 & 0,0436 & * & 0,221 & 0,0447 & $*$ & 0,221 & 0,0455 & "* & 0,238 & 0,0495 & $*$ & 0,24 & 0,0499 & $*$ & 0,238 & 0,05 \\
\hline Total sales in $t-1$ & & & & $-3 \mathrm{E}-07$ & $1,2 \mathrm{E}-07$ & " & $-3 \mathrm{E}-07$ & $1,2 \mathrm{E}-07$ & . & $-3 \mathrm{E}-07$ & $1,2 \mathrm{E}-07$ & 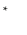 & $-3 \mathrm{E}-07$ & $1,6 \mathrm{E}-07$ & 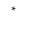 & $-3 \mathrm{E}-07$ & $1,6 \mathrm{E}-07$ \\
\hline Number of workers in $t-1$ & & & & 0,00105 & 0,00085 & & 0,00084 & 0,00095 & & 0,00084 & 0,00101 & & 0,00082 & 0,00102 & & 0,00074 & 0,00112 \\
\hline Exports in $t-1$ & & & & 0,287 & 0,223 & & 0,277 & 0,233 & & 0,242 & 0,241 & & 0,236 & 0,242 & & 0,254 & 0,245 \\
\hline Agricultural and forestry sector & & & & & & & $-0,131$ & 0,225 & & $-0,159$ & 0,234 & & $-0,162$ & 0,234 & & $-0,165$ & 0,235 \\
\hline Fishing sector & & & & & & & , & , & & , & , & & , & , & & , & , \\
\hline Mining sector & & & & & & & , & , & & , & , & & , & , & & , & , \\
\hline Manufacturing sector & & & & & & & $-0,516$ & 0,21 & * & $-0,562$ & 0,217 & ** & $-0,572$ & 0,218 & $"$ & $-0,583$ & 0,219 \\
\hline Electricity, gas and water sector & & & & & & & , & , & & , & , & & , & , & & , & , \\
\hline Construction sector & & & & & & & $-0,6$ & 0,327 & & $-0,625$ & 0,338 & & $-0,633$ & 0,339 & & $-0,642$ & 0,34 \\
\hline Commerce sector & & & & & & & $-0,768$ & 0,303 & * & $-0,773$ & 0,309 & * & $-0,775$ & 0,309 & & $-0,785$ & 0,309 \\
\hline Transport sector & & & & & & & $-1,021$ & 0,405 & 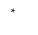 & $-1,05$ & 0,412 & . & $-1,053$ & 0,412 & & $-1,048$ & 0,413 \\
\hline Financial Services sector & & & & & & & , & , & & , & , & & , & , & & , & , \\
\hline Real estate sector & & & & & & & $-0,321$ & 0,2 & & $-0,34$ & 0,207 & & $-0,344$ & 0,207 & & $-0,338$ & 0,208 \\
\hline Social and health services sector & & & & & & & $-0,477$ & 0,323 & & $-0,531$ & 0,333 & & $-0,537$ & 0,332 & & $-0,547$ & 0,334 \\
\hline Other sectors & & & & & & & $-0,387$ & 0,308 & & $-0,39$ & 0,314 & & $-0,397$ & 0,314 & & $-0,406$ & 0,314 \\
\hline Tarapacá Region & & & & & & & & & & 3,607 & 191,5 & & 3,549 & 166,4 & & 3,555 & 164,4 \\
\hline Antofagasta Region & & & & & & & & & & 3,091 & 191,5 & & 3,033 & 166,4 & & 3,044 & 164,4 \\
\hline Atacama Region & & & & & & & & & & , & , & & , & , & & , & , \\
\hline Coquimbo Region & & & & & & & & & & 3,016 & 191,5 & & 2,961 & 166,4 & & 2,955 & 164,4 \\
\hline Valparaíso Region & & & & & & & & & & 3,457 & 191,5 & & 3,401 & 166,4 & & 3,406 & 164,4 \\
\hline O’Higgins Region & & & & & & & & & & 3,045 & 191,5 & & 2,985 & 166,4 & & 2,987 & 164,4 \\
\hline Maule Region & & & & & & & & & & 3,795 & 191,5 & & 3,734 & 166,4 & & 3,748 & 164,4 \\
\hline Bío Bío Region & & & & & & & & & & 3,303 & 191,5 & & 3,239 & 166,4 & & 3,242 & 164,4 \\
\hline Araucania Region & & & & & & & & & & 3,396 & 191,5 & & 3,337 & 166,4 & & 3,342 & 164,4 \\
\hline Los Lagos Region & & & & & & & & & & 2,947 & 191,5 & & 2,886 & 166,4 & & 2,89 & 164,4 \\
\hline Aysén Region & & & & & & & & & & 3,665 & 191,5 & & 3,605 & 166,4 & & 3,604 & 164,4 \\
\hline Magallanes Region & & & & & & & & & & 3,599 & 191,5 & & 3,54 & 166,4 & & 3,544 & 164,4 \\
\hline Metropolitan Region & & & & & & & & & & 3,436 & 191,5 & & 3,378 & 166,4 & & 3,384 & 164,4 \\
\hline Los Rios Region & & & & & & & & & & , & , & & , & , & & , & , \\
\hline Small firm & & & & & & & & & & & & & $-0,0953$ & 0,177 & & $-0,0974$ & 0,178 \\
\hline Private property & & & & & & & & & & & & & & & & $-0,0886$ & 0,754 \\
\hline Foreign Property & & & & & & & & & & & & & & & & , & , \\
\hline Mixed Property & & & & & & & & & & & & & & & & , & , \\
\hline Constant & $-2,238$ & 0,06 & * & $-2,112$ & 0,0766 & 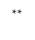 & $-1,701$ & 0,166 & " & $-5,092$ & 191,5 & & $-4,939$ & 166,4 & & $-4,845$ & 164,4 \\
\hline Observations & 3280 & & & 3280 & & & 3076 & & & 2925 & & & 2925 & & & 2862 & \\
\hline Pseudo R2 & 0,094 & & & 0,113 & & & 0,144 & & & 0,167 & & & 0,167 & & & 0,167 & \\
\hline
\end{tabular}

Source: Own elaboration. Note: $\left(^{*}\right)$ significant at $5 \%$ and $\left({ }^{* *}\right)$ significant at $1 \%$.

To contrast the previous results, a binary choice model using pseudo-panel data is estimated. Table 7 shows that the expenditure on introduction of innovations to the market in previous year and exports in previous year have a positive, significant and robust effect on the probability of obtaining public financing. At sectoral level, it is observed that the real state sector also has a positive, significant and robust effect. However, the expenditures on introduction of innovations to the market and external knowledge in previous year have no robust effects in magnitude, sign and / or statistical significance.

ISSN: 0718-2724. (http://jotmi.org) 
Table 7. Results with pseudo-panel data from innovation surveys

\begin{tabular}{|c|c|c|c|c|c|c|c|c|c|c|c|c|c|c|c|c|c|c|}
\hline & \multicolumn{2}{|c|}{ Model 1} & & \multicolumn{2}{|c|}{ Model 2} & & \multicolumn{2}{|c|}{ Model 3} & \multicolumn{3}{|c|}{ Model 4} & & \multicolumn{2}{|c|}{ Model 5} & \multicolumn{4}{|c|}{ Model 6} \\
\hline & Coef. & St. Error & & Coef. & $\begin{array}{l}\text { St. } \\
\text { Error }\end{array}$ & & Coef. & St. Error & & Coef. & $\begin{array}{l}\text { St. } \\
\text { Error }\end{array}$ & & Coef. & St. Error & & Coef. & $\begin{array}{l}\text { St. } \\
\text { Error }\end{array}$ & \\
\hline $\begin{array}{l}\text { Expenditure on equipment for } \\
\text { innovation in } t-1\end{array}$ & $2,6 \mathrm{E}-07$ & 4,7E-07 & & $-9 \mathrm{E}-07$ & $5 \mathrm{E}-07$ & & $-3 \mathrm{E}-07$ & $5,5 \mathrm{E}-07$ & & $-7 \mathrm{E}-07$ & 7,3E-07 & & $-8 \mathrm{E}-07$ & 7,9E-07 & & $-4 \mathrm{E}-07$ & $9,3 \mathrm{E}-07$ & \\
\hline $\begin{array}{l}\text { Expenditure on external knowl- } \\
\text { edge in } t-1\end{array}$ & $-3 \mathrm{E}-07$ & $3,8 \mathrm{E}-06$ & & $6,1 \mathrm{E}-06$ & $4 \mathrm{E}-06$ & & $1,3 \mathrm{E}-05$ & $4,8 \mathrm{E}-06$ & " & $1,4 \mathrm{E}-05$ & $6,1 \mathrm{E}-06$ & • & $1,4 \mathrm{E}-05$ & $6,2 \mathrm{E}-06$ & * & 1,7E-05 & $7,2 \mathrm{E}-06$ & * \\
\hline $\begin{array}{l}\text { Expenditure on training for } \\
\text { innovation in } t-1\end{array}$ & $3,8 \mathrm{E}-06$ & $9,8 \mathrm{E}-07$ & "* & $6,4 \mathrm{E}-06$ & $1,7 \mathrm{E}-06$ & ${ }^{*}$ & $5,4 \mathrm{E}-06$ & $1,9 \mathrm{E}-06$ & ${ }^{* *}$ & $3,1 \mathrm{E}-06$ & $2,3 \mathrm{E}-06$ & & $3,6 \mathrm{E}-06$ & 2,7E-06 & & $2,1 \mathrm{E}-06$ & $2,9 \mathrm{E}-06$ & \\
\hline $\begin{array}{l}\text { Expense on introd. of innovations } \\
\text { to the market } \mathrm{t}-1\end{array}$ & 1,9E-05 & $4,2 \mathrm{E}-06$ & $"$ & $2,6 \mathrm{E}-05$ & 4,4E-06 & $*$ & $1,5 \mathrm{E}-05$ & $4,9 \mathrm{E}-06$ & $*$ & $2,1 \mathrm{E}-05$ & 7,7E-06 & $"$ & $2,1 \mathrm{E}-05$ & 7,7E-06 & $"$ & $2 \mathrm{E}-05$ & $8,1 \mathrm{E}-06$ & . \\
\hline $\begin{array}{l}\text { Expenditure on other innovation } \\
\text { activities in } t-1\end{array}$ & $5,9 \mathrm{E}-05$ & $1,2 \mathrm{E}-05$ & $*$ & $-3 \mathrm{E}-05$ & $2 \mathrm{E}-05$ & & $-5 \mathrm{E}-05$ & $2,2 \mathrm{E}-05$ & . & $-9 \mathrm{E}-05$ & $3,1 \mathrm{E}-05$ & ${ }^{*}$ & $-8 \mathrm{E}-05$ & $3,6 \mathrm{E}-05$ & * & $-9 \mathrm{E}-05$ & $4 \mathrm{E}-05$ & . \\
\hline $\begin{array}{l}\text { No. of Intellectual property } \\
\text { rights in } t-1\end{array}$ & 0,00201 & 0,0159 & & 0,0102 & 0,0162 & & 0,019 & 0,0204 & & 0,0384 & 0,0303 & & 0,0361 & 0,0309 & & 0,036 & 0,0315 & \\
\hline Total sales in $\mathrm{t}-1$ & & & & $-1 \mathrm{E}-07$ & $4,1 \mathrm{E}-08$ & $*$ & $-1 \mathrm{E}-07$ & $4,3 \mathrm{E}-08$ & $*$ & $-9 \mathrm{E}-08$ & $5,2 \mathrm{E}-08$ & & $-1 \mathrm{E}-07$ & 6,7E-08 & & $-7 \mathrm{E}-08$ & $7,3 \mathrm{E}-08$ & \\
\hline Number of workers in $t-1$ & & & & 0,00398 & 0,00105 & $*$ & 0,00355 & 0,00122 & $*$ & 0,00459 & 0,00215 & . & 0,00396 & 0,00278 & & 0,00324 & 0,00306 & \\
\hline Exports in $\mathrm{t}-1$ & & & & 3,555 & 0,583 & $"$ & 5,936 & 0,899 & " & 5,658 & 1,365 & * & 5,393 & 1,557 & $*$ & 8,431 & 2,32 & " \\
\hline Agricultural and forestry sector & & & & & & & 0,0424 & 0,137 & & 0,37 & 0,242 & & 0,404 & 0,26 & & $-0,0659$ & 0,349 & \\
\hline Fishing sector & & & & & & & $-0,478$ & 0,308 & & $-0,464$ & 0,426 & & $-0,467$ & 0,423 & & $-0,951$ & 0,508 & \\
\hline Mining sector & & & & & & & $-0,827$ & 3,342 & & $-17,18$ & 22,09 & & $-15,1$ & 22,72 & & $-6,24$ & 23,44 & \\
\hline Manufacturing sector & & & & & & & $-0,215$ & 0,0839 & * & $-0,0342$ & 0,209 & & $-0,0426$ & 0,209 & & $-0,61$ & 0,339 & \\
\hline Electricity, gas and water sector & & & & & & & 0,144 & 0,723 & & $-0,595$ & 0,9 & & $-0,784$ & 1,066 & & 1,418 & 1,714 & \\
\hline Construction sector & & & & & & & 0,423 & 0,171 & * & 0,408 & 0,27 & & 0,364 & 0,297 & & 0,313 & 0,3 & \\
\hline Commerce sector & & & & & & & 0,0123 & 0,143 & & 0,114 & 0,31 & & 0,0901 & 0,314 & & $-0,224$ & 0,337 & \\
\hline Transport sector & & & & & & & $-0,25$ & 0,165 & & $-0,08$ & 0,297 & & $-0,105$ & 0,303 & & $-0,19$ & 0,33 & \\
\hline Financial Services sector & & & & & & & $-0,201$ & 0,369 & & $-0,133$ & 0,54 & & $-0,158$ & 0,559 & & 0,116 & 0,625 & \\
\hline Real estate sector & & & & & & & 0,472 & 0,0874 & $*$ & 0,544 & 0,187 & * & 0,517 & 0,201 & * & 0,619 & 0,215 & * \\
\hline Social and health services sector & & & & & & & 0,148 & 0,223 & & 0,544 & 0,364 & & 0,523 & 0,366 & & 0,915 & 0,555 & \\
\hline Other sectors & & & & & & & 0,174 & 0,305 & & 0,554 & 0,43 & & 0,558 & 0,429 & & 0,472 & 0,453 & \\
\hline Tarapacá Region & & & & & & & & & & 1,204 & 3,158 & & 0,446 & 3,829 & & 0,848 & 4,033 & \\
\hline Antofagasta Region & & & & & & & & & & $-4,191$ & 3,256 & & $-3,566$ & 3,705 & & $-0,811$ & 4,088 & \\
\hline Atacama Region & & & & & & & & & & 3,688 & 2,94 & & 3,269 & 3,162 & & $-1,698$ & 5,426 & \\
\hline Coquimbo Region & & & & & & & & & & 6,787 & 4,878 & & 7,976 & 5,942 & & $-0,5$ & 7,962 & \\
\hline Valparaíso Region & & & & & & & & & & $-3,453$ & 1,929 & & $-3,833$ & 2,222 & & $-2,453$ & 2,645 & \\
\hline O'Higgins Region & & & & & & & & & & $-0,533$ & 2,792 & & $-0,831$ & 2,907 & & $-1,97$ & 3,59 & \\
\hline Maule Region & & & & & & & & & & $-2,642$ & 2,802 & & $-2,4$ & 2,878 & & $-2,343$ & 3,285 & \\
\hline Bío Bío Region & & & & & & & & & & $-0,404$ & 1,514 & & $-0,479$ & 1,523 & & 1,509 & 1,85 & \\
\hline Araucania Region & & & & & & & & & & 2,609 & 1,86 & & 2,869 & 2,006 & & 6,002 & 2,64 & $\cdot$ \\
\hline Los Lagos Region & & & & & & & & & & $-0,714$ & 1,585 & & $-0,758$ & 1,577 & & $-1,907$ & 1,788 & \\
\hline Aysén Region & & & & & & & & & & 1,738 & 3,766 & & 1,736 & 3,749 & & 4,415 & 4,134 & \\
\hline Magallanes Region & & & & & & & & & & $-5,771$ & 3,175 & & $-6,092$ & 3,29 & & $-5,645$ & 4,07 & \\
\hline Metropolitan Region & & & & & & & & & & 0,0519 & 0,36 & & 0,0444 & 0,36 & & 0,0646 & 0,379 & \\
\hline Los Rios Region & & & & & & & & & & 1,7 & 3,416 & & 1,654 & 3,394 & & 2,75 & 3,893 & \\
\hline Small firm & & & & & & & & & & & & & $-0,341$ & 0,981 & & $-0,16$ & 1,155 & \\
\hline Private property & & & & & & & & & & & & & & & & 11,45 & 8,634 & \\
\hline Foreign Property & & & & & & & & & & & & & & & & $-1,775$ & 10,34 & \\
\hline Mixed Property & & & & & & & & & & & & & & & & 15,51 & 10,56 & \\
\hline Constant & $-1,978$ & 0,0317 & 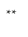 & $-2,224$ & 0,072 & $"$ & $-2,392$ & 0,0978 & ${ }^{*}$ & $-2,319$ & 0,25 & $"$ & $-2,049$ & 0,813 & * & $-13,56$ & 8,524 & \\
\hline Observations & 12039 & & & 12039 & & & 12039 & & & 12039 & & & 12039 & & & 12039 & & \\
\hline Pseudo R2 & 0,016 & & & 0,029 & & & 0,044 & & & 0,053 & & & 0,053 & & & 0,055 & & \\
\hline
\end{tabular}

Source: Own elaboration. Note: $\left({ }^{*}\right)$ significant at $5 \%$ and $\left({ }^{* *}\right)$ significant at $1 \%$

ISSN: 0718-2724. (http://jotmi.org) 


\section{Conclusions}

It is possible to conclude, using pseudo-panels and cross-sectional data, that policymakers and members of evaluating committees follow a strategy of "picking the winner" because small and medium sized firms that have some type of expenditures on innovation activities in previous year are more likely to obtain public support for innovation.

By using pseudo-panels, it is observed that firms with expenditure on introduction of innovations to the market in previous year and exports in previous year have more probability to obtain public support for innovation. On the other hand, with cross-sectional data, there are different expenditures on innovation activities in previous year that affect the probability of obtaining public support, depending of the version of Innovation Survey.

When comparing the above results with the international literature, it is observed that the factors that explain the allocation of public funds for innovation in other countries are more diverse than those observed in the case of Chile, which leads to the conclusion that perhaps the award criteria of these funds should be changed to guide the earlier innovative actions of the applicant firms.

\section{References}

Afcha S. (2012). Analyzing the interaction between R\&D subsidies and firm's innovation strategy. Journal of Technology Management \& Innovation, 7, 57-70. doi: 10.4067/s0718-27242012000300006

Álvarez, R., Bravo-Ortega, C., \& Navarro, L. (2010). Innovation, R\&D Investment and Productivity in Chile. SSRN Electronic Journal. doi:10.2139/ssrn. 1818741

Álvarez, R., Bravo-Ortega, C. \& Zahler, A. (2015). Innovation and Productivity in Services: Evidence from Chile. Emerging Markets Finance and Trade, 51(3), 593-611. doi:10.1080/1540496X.2015.1026696

Barge-Gil, A., \& López, A. (2014). R\&D determinants: Accounting for the differences between research and development. Research Policy, 43(9), 1634-1648. doi:10.1016/j.respol.2014.04.017

Benavente J. (2005). Investigación y desarrollo, innovación y productividad: un análisis econométrico a nivel de la firma. Estudios de Economía, 32, 39-67

Bravo-Ortega, C., Benavente, J. M. \& González, A. 2014. Innovation, exports and productivity: learning and self-selection in Chile. Emerging Markets Finance and Trade, 50 (1), 68-95. doi:10.2753/REE1540496X5001S105

Cabaleiro, G. \& Salce, F. (2018). The State of Markets for Technology in Chile. Journal of Technology Management \& Innovation, 13(1), 3847. doi:10.4067/S0718-27242018000100038

Canales, M., \& Álvarez, R. (2017). Impacto de los obstáculos al conocimiento en la innovación de las empresas chilenas. Journal of Technology Management \& Innovation, 12(3), 78-85. doi:10.4067/S0718-27
Cantner, U. \& Kösters, S. (2012). Picking the winner? Empirical evidence on the targeting of R\&D subsidies to start-ups. Small Business Economics, 39(4), 921-936. doi:10.1007/s11187-011-9340-9

Cerulli, G., \& Potí, B. (2012). Evaluating the robustness of the effect of public subsidies on firms' R\&D: an application to Italy. Journal of Applied Economics, 15(2), 287-320. doi:10.1016/S1514-0326(12)60013-0

Clausen, T. (2009). Do subsidies have positive impacts on R\&D and innovation activities at the firm level? Structural Change and Economic Dynamics, 20(4), 239-253. doi:10.1016/j.strueco.2009.09.004

Crespi, G., Figal, L., Maffioli, A. \& Meléndez, M. (2015). Long-Term Productivity Effects of Public Support to Innovation in Colombia. Emerging Markets Finance and Trade, 51(1), 1-17. doi:10.1080/1540 496X.2015.998080

Crespi, G., Tacsir, E., \& Vargas, F. (2016). Innovation Dynamics and Productivity: Evidence for Latin America. Firm Innovation and Productivity in Latin America and the Caribbean, 37-71. doi:10.1057/978-1-349-58151-1_2

Deaton, A. (1985). Panel Data from Time Series of Cross Sections. Journal of Econometrics, 30(1-2), 109-126. doi:10.1016/03044076(85)90134-4242017000300008

Duch-Brown, N., Garcia-Quevedo, J., \& Montolio, D. (2011). The Link between Public Support and Private R\&D Effort: What is the Optimal Subsidy? SSRN Electronic Journal. doi:10.2139/ssrn.1864192

González, X., \& Pazó, C. (2008). Do public subsidies stimulate private R\&D spending? Research Policy, 37(3), 371-389. doi:10.1016/j.respol.2007.10.009

Huergo E., Trenado M., \& Ubierna A. (2016). The impact of public support on firm propensity to engage in R\&D: Spanish experience. Technological Forecasting \& Social Change, 113(B), 206-219. doi:10.1016/j.techfore.2015.05.011

Moffitt, R. (1993). Identification and Estimation of Dynamic Models with a Time Series of Repeated Cross-Sections. Journal of Econometrics, 59, 99-123. doi:10.1016/0304-4076(93)90041-3

Moya, P. \& Molina, F. (2017). Innovación y Emprendimiento en el Discurso Político Chileno. Journal of Technology Management \& Innovation, 12(1), 93-99. doi:10.4067/S0718-27242017000100010

Shefer, D., \& Frenkel, A. (2005). R\&D, firm size and innovation: an empirical analysis. Technovation, 25(1), 25-32. doi:10.1016/S01664972(03)00152-4

Verbeek M., \& Vella F. (2005). Estimating dynamic models from repeated cross-sections. Journal of Econometrics, 127, 83-102. doi:10.1016/j.jeconom.2004.06.004

Verbeek M. (2008). Pseudo-Panels and repeated Cross-Sections. In The Econometrics of Panel Data. Springer, third edition, pp. 369-383. doi:10.1007/978-3-540-75892-1_11 
